\title{
Cross-Cultural Religious Tourism with Impression Distance Search System
}

\author{
Piyaporn NURARAK ${ }^{\mathrm{a}, 1}$, Shiori SASAKI ${ }^{\mathrm{a}}$, Irene Erlyn Wina RACHMAWAN ${ }^{\mathrm{a}}$ and \\ Yasushi KIYOKI ${ }^{\mathrm{a}}$
}

${ }^{\mathrm{a}}$ Graduate School of Media and Governance, Keio University, Japan

\begin{abstract}
Cross-cultural religious tourism is computational to promote crosscultural communication and understanding according to impression distance. Our motivation to implement semantic search with an emotion-oriented context into the proposed system is to realize global tourism recommendations expressed in different cultures. The objectives of this paper are (1) to find the religious places by using the tourist's emotional distance, (2) to find similar religious places not only in the same culture but also in the different cultures with the tourist's emotional distance calculations. Experimental results demonstrate the feasibility and applicability of this method.
\end{abstract}

Keywords. Cross-cultural, Impression distance, Religious, Tourism.

\section{Introduction}

Nowadays, from a quantitative perspective, and in general terms, Tourism Industry is one of the economic sectors that is growing increasingly all over the world, according to the United Nations World Tourism Organization (UNWTO)[1]. Low-cost travels make new tourism industry. In several globalized social phenomena, people across the world can easily exchange ideas and values whether they are in different environmental, technological, cultural, or economic settings. Consequently, this exchange has enabled the circulation of massive knowledge amongst people to understand one another better and this has hugely boosted tourism.

We aim to recognize an emotional context in religious places. We provide a function for tourists to find impressions for tourist places. Numerous studies have proposed various methods for information retrieval using "Kansei" and semantic computing. Semantic computing is an important approach to analysis and information retrieval. The function using emotion finds the essential features related to an impression context, assisting tourists to find the desired places. The described of the Mathematical Model of Meaning (MMM) [2] [3] for emotion-based information retrieval and emotional based retrieval system. MMM has proposed a meta-database knowledge system with a new search method of semantic associative [2] [3]. In those studies, semantic-searching in sensibility and measuring the impression were realized in semantic spaces. In the Mathematical Model of Meaning [2] [3] [4] [5], an orthogonal semantic space is created

\footnotetext{
${ }^{1}$ Corresponding Author, Graduate School of Media and Governance, Keio University, Shonan Fujisawa Campus, 5322 Endo, Fujisawa, Kanagawa, 252-0882, Japan; Email: pnurarak@sfc.keio.ac.jp.
} 
for semantic associative search. In the impression distance in semantic search system, our method is using a vector space and realizes calculations on relationships between place-data mapped in semantic space and emotion.

This paper is organized as follows: Section 2 shows the overview of our impression distance search system for cross-cultural religious tourism and its key technologies. Section 3 shows the implementation of a prototype system. Section 4 presents our experimental results of the implemented prototype system, and the conclusion and future work in section 5 .

\section{Impression Distance Search System}

This paper proposes cross-cultural religious tourism, with computations for promoting cross-cultural communication and understanding according to impression distance. Our motivation to implement a semantic search with emotion-oriented contexts is to make global tourism recommendations expressed in different cultures. The objectives of this paper are (1) to realize a system to find the religious places by using the tourist's emotional distance, (2) to realize a system to find similar religious places not only in the same culture but also in the different cultures that have the same tourist's emotion.

\subsection{Creation of Kansei Spaces}

A Kansei space of religious tourism is connected for impression distance. A Kansei vector space $\mathrm{K}$ is created as $\mathrm{p}+4$ dimensions by the impression which expresses religious place name, image, latitude, longitude, and $\mathrm{p}$ impression words of religious tourism as shown in Figure 1. The matrix is defined as Kensei vector space $\mathrm{K}_{\mathrm{i}}$, when $\mathrm{i}$ define cultural $(\mathrm{i}=1,2,3, . . \mathrm{m})$. In the row, vector data $T_{i j}(j=1,2, \ldots, n)$ composed and their impressions are registered.



Figure 1. A Kansei-Tour Vector Space 


\subsection{Cosine Similarity}

Cosine similarity measures the cosine of the angle between two non-zero vectors of an inner product space. This similarity measurement is particularly concerned with orientation, rather than magnitude. In short, two cosine vectors that are aligned in the same orientation will have a similarity measurement of 1 , whereas two vectors aligned perpendicularly will have a similarity of 0 . If two vectors are diametrically opposed, meaning they are oriented in exactly opposite directions (i.e. back-to-back), then the similarity measurement is -1 . Often, however, cosine similarity is used in positive space, between the bounds 0 and 1 . Cosine similarity is not concerned, does not measure, differences is the magnitude and is only a representation of similarities in orientation ([6] [7]).

The Cosine similarity measurement begins by finding the cosine of the two non-zero vectors. This can be derived using the Euclidean dot product formula which is written as:

$$
A \cdot B=\|A\|\|B\| \cos \theta
$$

Then, given the two vectors and the dot product, the cosine similarity is defined as:

$$
\text { Cosine Similarity }=\cos \theta=\frac{A \cdot B}{\|A\|\|B\|}=\frac{\sum_{i=1}^{n} A_{i} B_{i}}{\sqrt{\sum_{i=1}^{n} A_{i}^{2}} \sqrt{\sum_{i=1}^{n} B_{i}^{2}}}
$$

In other words, the cosine of the angle between two vectors is the inner product of the vectors, after they have been normalized to unit length. If A and B are frequency vectors for impressions, a higher relate impression will have a long vector and a rarely relate impression will have a short vector, yet the impression might be synonyms. Cosine captures the idea that the length of the vectors is irrelevant; the important thing is the angle between the vectors. The cosine ranges from -1 when the vectors point in opposite directions ( $\theta$ is 180 degrees) to +1 when they point in the same direction ( $\theta$ is 0 degrees). When the vectors are orthogonal ( $\theta$ is 90 degrees), the cosine is zero. With raw impression vectors, which necessarily cannot have negative elements, the cosine cannot be negative, but weighting and smoothing often introduce negative elements.

\section{Implementation of a prototype system}

To recognize an emotion context, the impression distance search system realizes a function for the users to express their impressions, such as interesting, clam, elegant, etc., for religious tourism places in the same culture or different cultures. This function finds the most essential features related to emotion contexts, given as the user's impression of 
the religious place queries. The overview of this system is shown in Figure 2. This system consists of 3 processes: (a) Data aggregation, (b) Space creation and integration and (c) Data retrieval and querying. The procedure of proposed idea is represented in Figure 2.

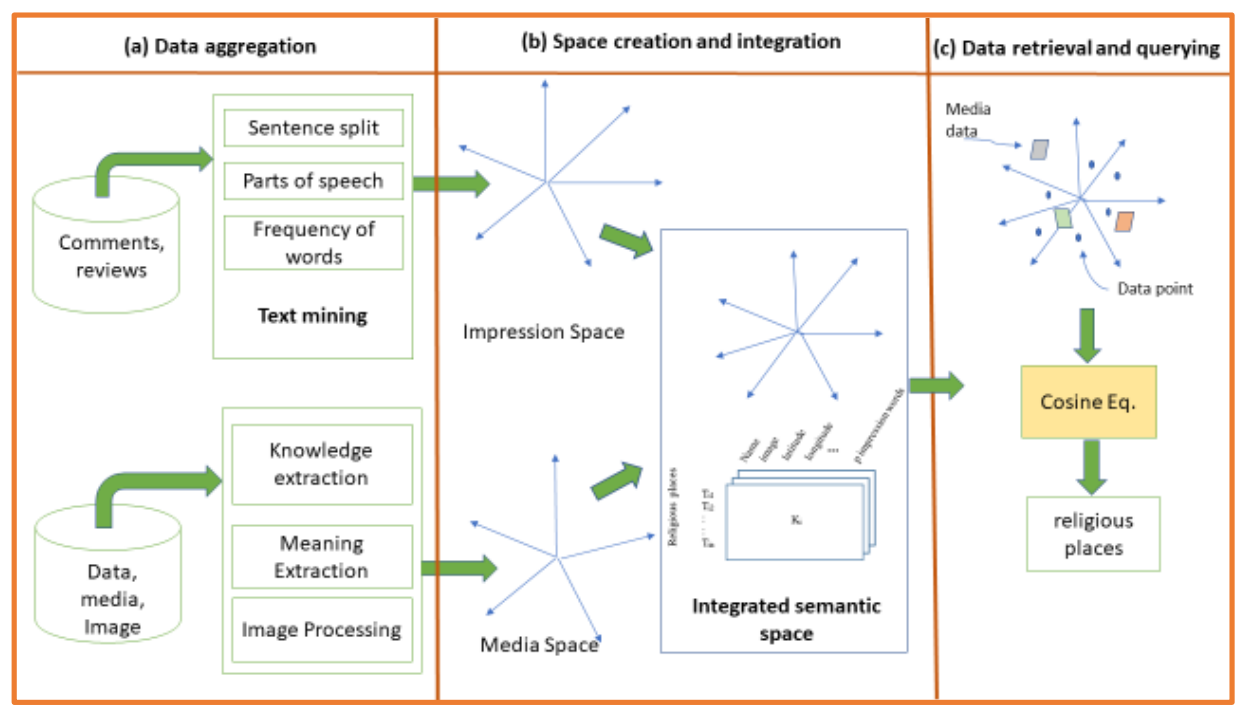

Figure 2. System overview and operating procedure.

\section{(a) Data aggregation}

In the process, we apply information on the religious places by using digital documents and traveler reviews from 48 travel websites. Then we using text mining, such as tf-idf to filtering and frequently words, to find impressions of the places. Moreover, the images of the places have been used to find the meaning of the places.

\section{(b) Space creation and integration}

After that, we select useful data to create impression space and media space and integrated both spaces into semantic space.

\section{(c) Data retrieval and querying}

We use cosine similarity to find the places that have the same impression or close to in the same culture or different culture by ranking..

\section{Experiment results}

To clarify the feasibility and applicability of our method, we have implemented our impression distance search system for temple datasets. In experimental study, we use 10 
Thailand temples located in Bangkok area, 10 Japan Temples located in Tokyo area, 10 Finland churches located in Helsinki area and 13 emotion contexts as dataset. We set retrieved result by ranking that can be (1) show the Thailand temples /Japan temples /Finland churches by using emotion context, (2) show the temples in the same religiouscultures that has the same emotion context and (3) show the temples in the other two different religious-cultures that have the same emotion context by queries.

\subsection{Experiment 1}

10 Thailand temples were collected and people were asked to judge the implied emotion of each temples. Table 1 is the result of impression words which were extracted from answers for Thailand temples. We also calculated the precision and recall to evaluate the retrieval performance of our method (illustrated in Table 2). The total precision is $63 \%$ and the total recall is $72 \%$.

Table 1. Experimental results of emotion detection for 10 Thailand temples

\begin{tabular}{cccccccccccccc}
\hline id & \multicolumn{10}{c}{} & \multicolumn{10}{c}{ Detected Emotion } & \multicolumn{1}{c}{$c$} \\
& C1 & C2 & C3 & C4 & C5 & C6 & C7 & C8 & C9 & C10 & C11 & C12 & C13 \\
\hline 1 & 0.3 & 0.2 & 0.2 & 0.8 & 0.4 & 0.7 & 0.4 & 0.5 & 0.5 & 0.8 & 0.4 & 0.3 & 0.4 \\
2 & 0.6 & 0.4 & 0.2 & 0.8 & 0.2 & 0.8 & 0.5 & 0.6 & 0.8 & 0.8 & 0.1 & 0.8 & 0.1 \\
3 & 0.5 & 0.4 & 0.1 & 0.3 & 0.5 & 0.4 & 0.6 & 0.8 & 0.3 & 0.8 & 0.1 & 0.8 & 0.1 \\
4 & 0.3 & 0.3 & 0.1 & 0.4 & 0.6 & 0.3 & 0.3 & 0.3 & 0.3 & 0.3 & 0.2 & 0.3 & 0.4 \\
5 & 0.2 & 0.1 & 0.3 & 0.3 & 0.6 & 0.1 & 0.4 & 0.3 & 0.3 & 0.4 & 0.6 & 0.3 & 0.2 \\
6 & 0.6 & 0.5 & 0.1 & 0.4 & 0.4 & 0.7 & 0.6 & 0.8 & 0.3 & 0.5 & 0.3 & 0.2 & 0.1 \\
7 & 0.4 & 0.2 & 0.3 & 0.3 & 0.5 & 1 & 0.6 & 0.6 & 0.9 & 0.5 & 0.2 & 0.8 & 0.1 \\
8 & 0.4 & 0.1 & 0.4 & 0.3 & 0.6 & 0.1 & 0.3 & 0.3 & 0.3 & 0.5 & 0.7 & 0.2 & 0.2 \\
9 & 0.1 & 0.1 & 0.2 & 0.3 & 0.4 & 0.4 & 0.2 & 0.1 & 0.3 & 0.6 & 0.4 & 0.1 & 0.4 \\
10 & 0.7 & 0.5 & 0.1 & 0.7 & 0.4 & 0.1 & 0.9 & 0.9 & 0.8 & 0.4 & 0.2 & 0.7 & 0.1 \\
\hline \hline
\end{tabular}

$\mathrm{C} 1=$ interesting, $\mathrm{C} 2=$ mysterious, $\mathrm{C} 3=$ rural-like, $\mathrm{C} 4=$ relaxed, $\mathrm{C} 5=$ calm, $\mathrm{C} 6=$ unique, $\mathrm{C} 7=$ elegant, $\mathrm{C} 8=$ precious, $\mathrm{C} 9=$ faithful, $\mathrm{C} 10=$ historical, $\mathrm{C} 11=$ quiet, $\mathrm{C} 12=$ bustling, $\mathrm{C} 13=$ commonplace.

Detected emotion means implied emotion of religious places which were detected by our system

Table 2. Retrieval performance for Thailand temples

\begin{tabular}{ccccccccccccccc}
\hline & C1 & C2 & C3 & C4 & C5 & C6 & C7 & C8 & C9 & C10 & C11 & C12 & C13 & Average \\
\hline $\begin{array}{c}\text { Precision } \\
(\%)\end{array}$ & 100 & 75 & 42.9 & 62.5 & 50 & 100 & 50 & 80 & 50 & 60 & 57.1 & 57.1 & 40 & 63.43 \\
Recall (\%) & 85.7 & 60 & 75 & 100 & 75 & 100 & 50 & 66.7 & 33.3 & 75 & 66.7 & 80 & 66.7 & 71.85 \\
\hline \hline
\end{tabular}

To start with Thailand culture, we select impression keyword as "calm", similarity places as "Wat Arun", Similarity Thai to Japanese as "Wat Pho" and Similarity Thai to Finland as "Wat Suthat", shown in Figure 3.

The impression distance search system shows the result of Thai temples that relate to this impression by queries. As shown in Figure 4, the closest to the impression of "calm" are "Wat Benjamabhopit", "Wat Traimit" and "Wat Suthat" that has 0.6 in similarity. On the other hand, "Wat Pho" has 0.2 that means this temple is not good place to visit if the tourist wants to calm. 


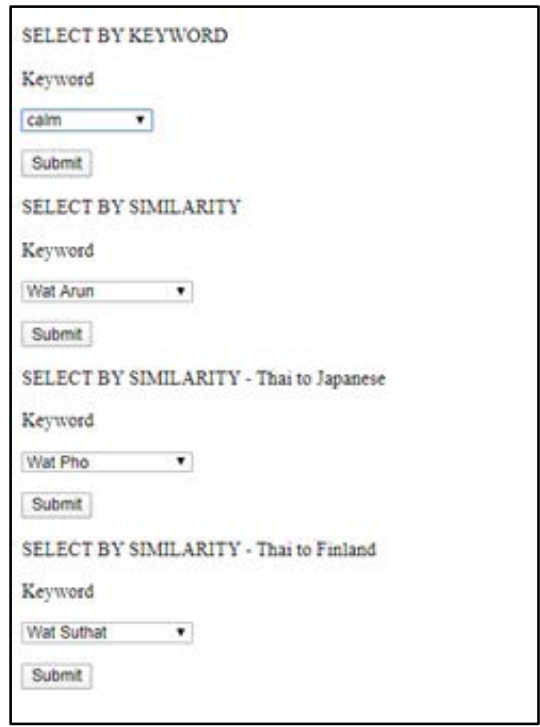

Figure 3. Selected impression and Thailand temples.

\section{Similar THAILAND TEMPLES by emotional keyword \\ calm}


Wat Pho, 0.2

Figure 4.Thailand temples by "clam" impression. 
Figure 5 shown Thailand temples that give the tourist feeling the same emotion as "Wat Arun" that means if tourist knows "Wat Arun" well and want to find the other temples in Bangkok that given the same impression with, this system give you the answer. Figure 6 presents Japan temples that give the tourists feeling same as "Wat Pho" in Thailand.

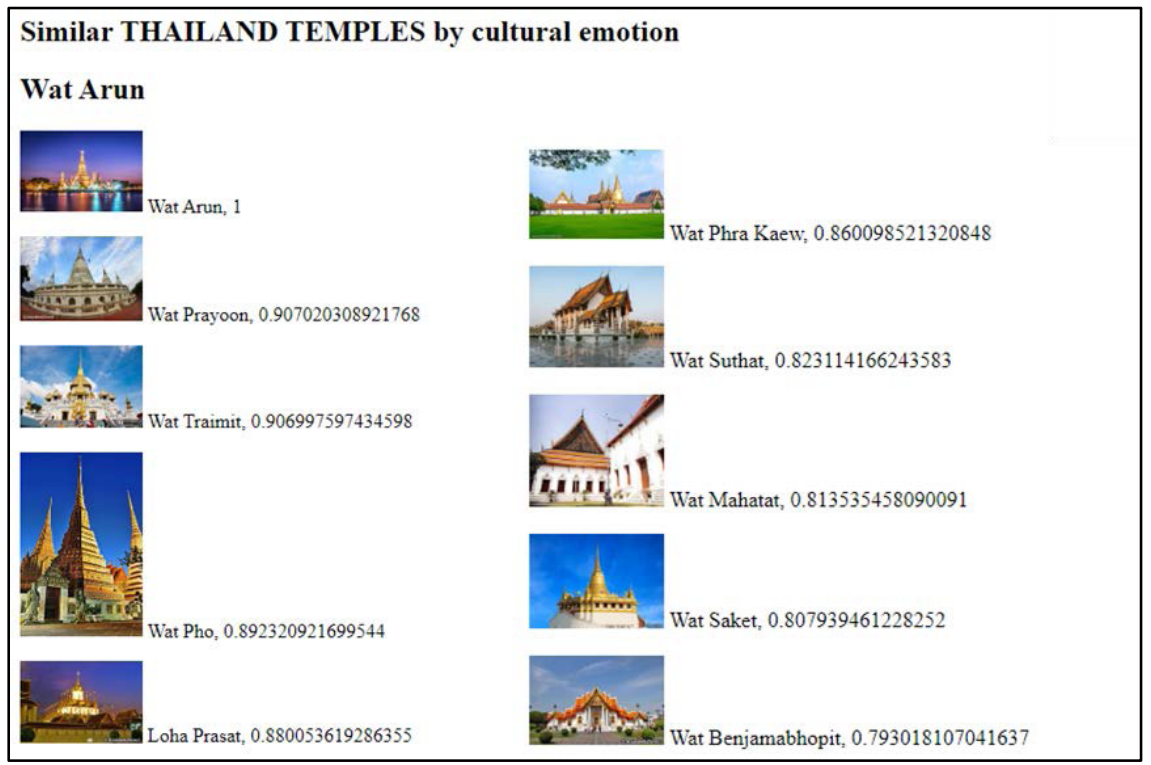

Figure 5. Search results of Thailand temples by similar to "Wat Arun" impression.



Figure 6. Japan temples by similar to "Wat Pho" impression. 
Figure 7. shows Finland Churches that have the same impression as "Wat Suthat". Even though Finland churches and Thailand temples are not the same religion, this system can find the emotional distance by using context.

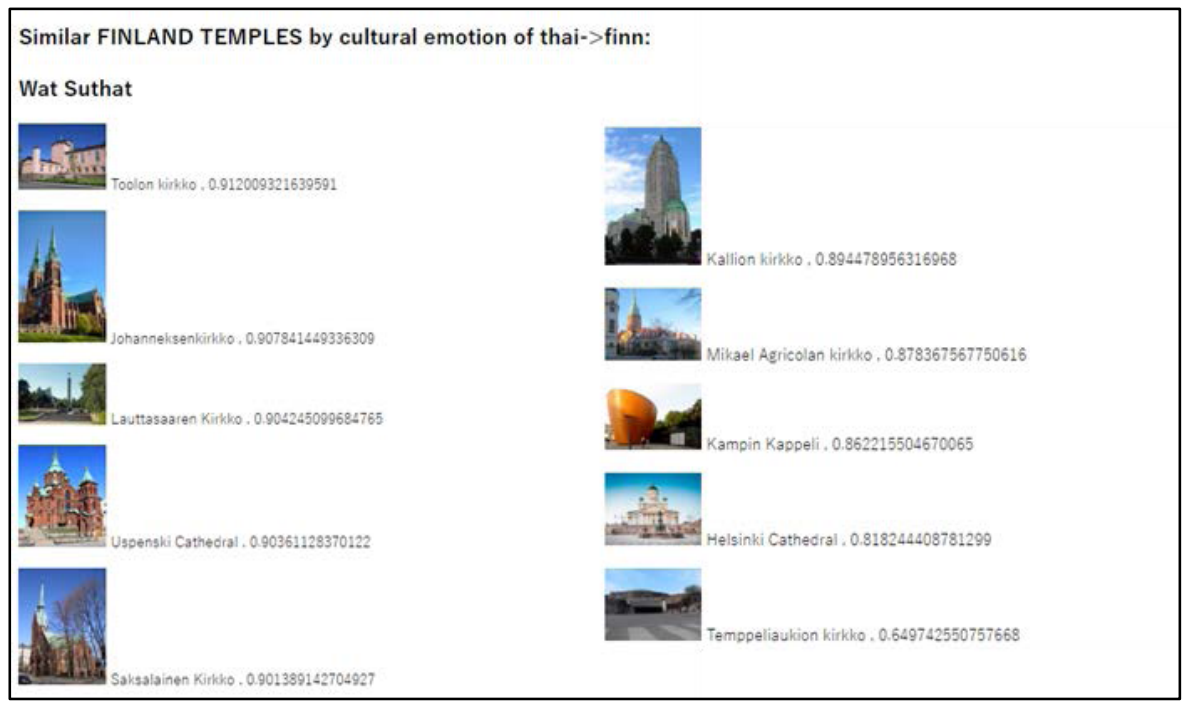

Figure 7. Search results of Finland churches in the same impression as "Wat Suthat".

\subsection{Experiment 2}

In a similar way, an experiment was done with Japan temples. The detail results of temples retrieval are shown in Table 3. The precision and recall of information retrieval are shown in Table 4 . The total precision is $70 \%$ and the total recall is $68 \%$.

Table 3. Experimental results of emotion detection for 10 Japan temples

\begin{tabular}{cccccccccccccc}
\hline id & \multicolumn{1}{c}{} & \multicolumn{10}{c}{ Detected Emotion } & \multicolumn{1}{c}{$c$} \\
& C1 & C2 & C3 & C4 & C5 & C6 & C7 & C8 & C9 & C10 & C11 & C12 & C13 \\
\hline 1 & 0.6 & 0.3 & 0.5 & 0.2 & 0.6 & 0.1 & 0.2 & 0.4 & 0.6 & 0.6 & 0.4 & 0.6 & 0.1 \\
2 & 0.3 & 0.1 & 0.1 & 0.2 & 0.1 & 0.5 & 0.2 & 0.3 & 0.6 & 0.2 & 0.1 & 0.8 & 0.3 \\
3 & 0.4 & 0.1 & 0.3 & 0.6 & 0.3 & 0.2 & 0.3 & 0.3 & 0.6 & 0.7 & 0.3 & 0.4 & 0.1 \\
4 & 0.5 & 0.1 & 0.7 & 0.6 & 0.8 & 0.9 & 0.2 & 0.3 & 0.8 & 0.5 & 0.8 & 0.3 & 0.2 \\
5 & 0.6 & 0.4 & 0.7 & 0.3 & 0.5 & 0.3 & 0.3 & 0.4 & 0.5 & 0.7 & 0.4 & 0.4 & 0.1 \\
6 & 0.2 & 0.1 & 0.3 & 0.4 & 0.1 & 0.3 & 0.1 & 0.1 & 0.5 & 0.2 & 0.1 & 0.1 & 0.3 \\
7 & 0.1 & 0.2 & 0.1 & 0.2 & 0.2 & 0.7 & 0.1 & 0.1 & 0.5 & 0.3 & 0.3 & 0.5 & 0.3 \\
8 & 0.4 & 0.1 & 0.5 & 0.6 & 0.7 & 0.3 & 0.3 & 0.2 & 0.6 & 0.5 & 0.2 & 0.5 & 0.1 \\
9 & 0.4 & 0.2 & 0.3 & 0.3 & 0.4 & 0.3 & 0.2 & 0.2 & 0.6 & 0.5 & 0.4 & 0.4 & 0.1 \\
10 & 0.3 & 0.5 & 0.5 & 0.3 & 0.2 & 0.3 & 0.1 & 0.3 & 0.5 & 0.6 & 0.5 & 0.1 & 0.1 \\
\hline
\end{tabular}

$\mathrm{C} 1=$ interesting, $\mathrm{C} 2=$ mysterious, $\mathrm{C} 3=$ rural-like, $\mathrm{C} 4=$ relaxed, $\mathrm{C} 5=$ calm, $\mathrm{C} 6=$ unique, $\mathrm{C} 7=$ elegant, $\mathrm{C} 8=$ precious, $\mathrm{C} 9=$ faithful, $\mathrm{C} 10=$ historical, $\mathrm{C} 11=$ quiet, $\mathrm{C} 12=$ bustling, $\mathrm{C} 13=$ commonplace.

Detected emotion means implied emotion of religious places which were detected by our system

Table 4. Retrieval performance for Japan temples

\begin{tabular}{ccccccccccccccc}
\hline & $\mathrm{C} 1$ & $\mathrm{C} 2$ & $\mathrm{C} 3$ & $\mathrm{C} 4$ & $\mathrm{C} 5$ & $\mathrm{C} 6$ & $\mathrm{C} 7$ & $\mathrm{C} 8$ & $\mathrm{C} 9$ & $\mathrm{C} 10$ & $\mathrm{C} 11$ & $\mathrm{C} 12$ & $\mathrm{C} 13$ & Average \\
\hline $\begin{array}{c}\text { Precision } \\
(\%)\end{array}$ & 100 & 40 & 40 & 100 & 100 & 100 & 33.33 & 28.57 & 100 & 75 & 60 & 75 & 60 & 70.15 \\
$\begin{array}{c}\text { Recall (\%) } \\
66.67\end{array}$ & 40 & 50 & 100 & 62.5 & 100 & 25 & 50 & 75 & 60 & 50 & 100 & 100 & 67.63 \\
\hline \hline
\end{tabular}


This experiment from the Japanese site, shows the Japanese temples by using an emotion context and using Japan temples. In addition, by select Japan temples will show Thailand temples and Finland churches that have the same impression distance. Figure 8 shows the selected emotion and Japan temples. Figure 9 show the result of "elegant" impression.

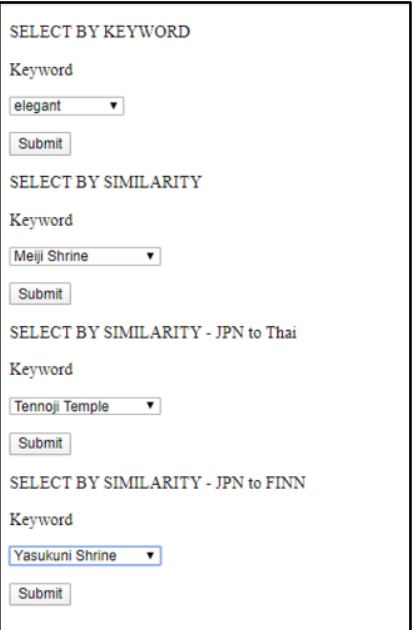

Figure 8. Selected impression and Japan temples.

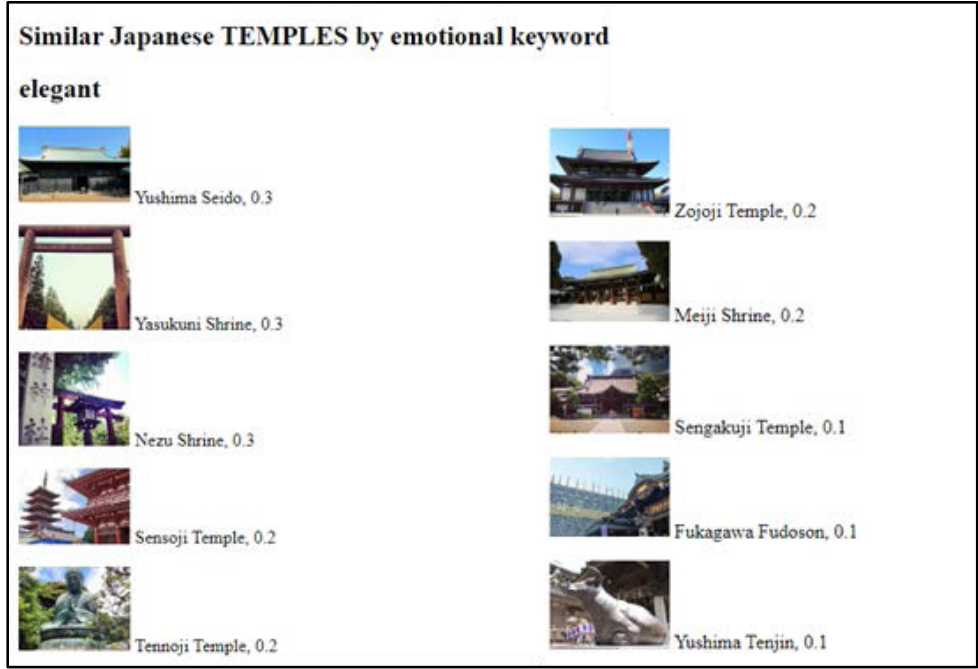

Figure 9. Japan temples with "elegant" impression.

Figure 10 shows Japan Temples that have the same emotion context with "Meiji shrine" and Thailand temples that have the same impression as "Tennoji temple" represent in Figure 11 


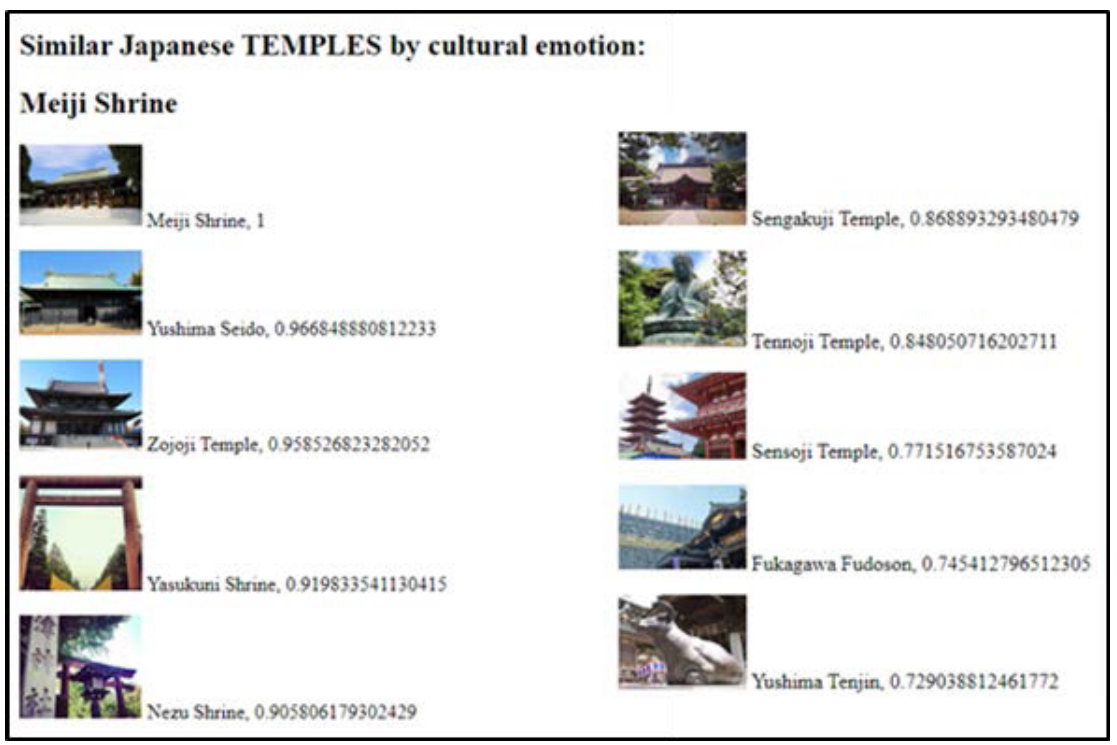

Figure 10. Search results of Japan temples with "Meiji shrine".



Figure 11. Thailand temples similarity with "Tennoji temple".

Figure 12 shows Finland churches that have impression distance close to "yasukuni shrine". 


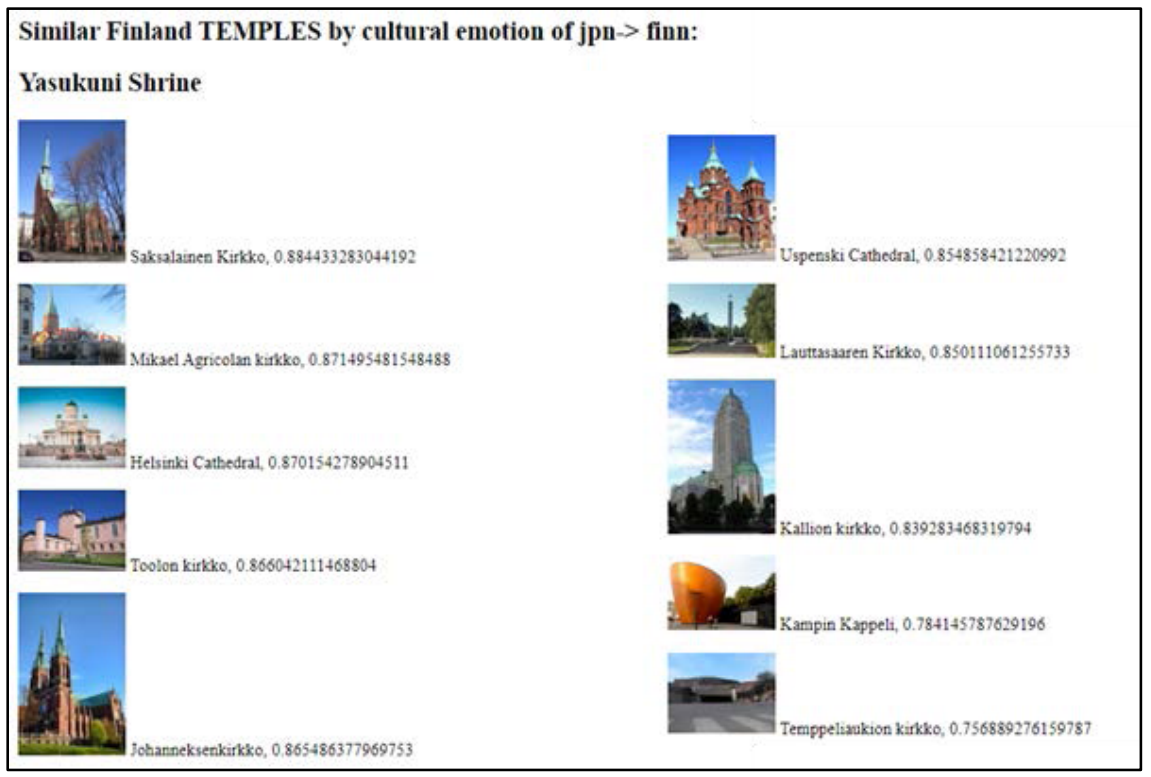

Figure 12. Finland churches similarity with "Yasukuni shrine".

\subsection{Experiment 3}

The similar tasks were done with Finland Churches as well. The detail results of emotion detection are shown in Table 5. In addition, the precision and recall were calculated as Table 6 . The total precision is $61 \%$ and the total recall is $75 \%$.

Table 5. Experimental results of emotion detection for 10 Finland churches

\begin{tabular}{|c|c|c|c|c|c|c|c|c|c|c|c|c|c|}
\hline \multirow{2}{*}{ id } & \multicolumn{13}{|c|}{ Detected Emotion } \\
\hline & $\mathrm{C} 1$ & $\mathrm{C} 2$ & C3 & $\mathrm{C} 4$ & $\mathrm{C} 5$ & $\mathrm{C} 6$ & $\mathrm{C} 7$ & $\mathrm{C} 8$ & $\mathrm{C9}$ & C10 & C11 & C12 & $\mathrm{C} 13$ \\
\hline 1 & 0.5 & 0.1 & 0.1 & 0.2 & 0.6 & 0.3 & 0.6 & 0.6 & 0.7 & 0.7 & 0.5 & 0.3 & 0.1 \\
\hline 2 & 0.5 & 0.1 & 0.1 & 0.5 & 0.5 & 0.9 & 0.6 & 0.6 & 0.8 & 0.6 & 0.4 & 0.5 & 0.1 \\
\hline 3 & 0.7 & 0.4 & 0.1 & 0.3 & 0.3 & 0.7 & 0.1 & 0.3 & 0.4 & 0.1 & 0.2 & 0.3 & 0.2 \\
\hline 4 & 0.3 & 0.2 & 0.2 & 0.3 & 0.4 & 0.3 & 0.6 & 0.6 & 0.7 & 0.6 & 0.5 & 0.4 & 0.1 \\
\hline 5 & 0.6 & 0.3 & 0.1 & 0.2 & 0.8 & 0.8 & 0.4 & 0.5 & 0.5 & 0.3 & 0.8 & 0.2 & 0.2 \\
\hline 6 & 0.5 & 0.2 & 0.1 & 0.3 & 0.4 & 0.3 & 0.5 & 0.5 & 0.4 & 0.3 & 0.4 & 0.2 & 0.2 \\
\hline 7 & 0.3 & 0.1 & 0.2 & 0.3 & 0.3 & 0.3 & 0.4 & 0.3 & 0.7 & 0.3 & 0.4 & 0.2 & 0.3 \\
\hline 8 & 0.2 & 0.1 & 0.3 & 0.3 & 0.3 & 0.3 & 0.3 & 0.2 & 0.5 & 0.2 & 0.5 & 0.2 & 0.4 \\
\hline 9 & 0.2 & 0.2 & 0.2 & 0.3 & 0.4 & 0.3 & 0.4 & 0.3 & 0.7 & 0.4 & 0.4 & 0.2 & 0.3 \\
\hline 10 & 0.4 & 0.1 & 0.1 & 0.2 & 0.4 & 0.3 & 0.4 & 0.4 & 0.5 & 0.4 & 0.4 & 0.2 & 0.2 \\
\hline
\end{tabular}

$\mathrm{C} 1=$ interesting, $\mathrm{C} 2=$ mysterious, $\mathrm{C} 3=$ rural-like, $\mathrm{C} 4=$ relaxed $, \mathrm{C} 5=\mathrm{calm}, \mathrm{C} 6=$ unique, $\mathrm{C} 7=$ elegant, $\mathrm{C} 8=$ precious, $\mathrm{C} 9=$ faithful, $\mathrm{C} 10=$ historical, $\mathrm{C} 11=$ quiet, $\mathrm{C} 12=$ bustling, $\mathrm{C} 13=$ commonplace.

Detected emotion means implied emotion of religious places which were detected by our system

Table 6. Retrieval performance for Finland churches

\begin{tabular}{ccccccccccccccc}
\hline & $\mathrm{C} 1$ & $\mathrm{C} 2$ & $\mathrm{C} 3$ & $\mathrm{C} 4$ & $\mathrm{C} 5$ & $\mathrm{C} 6$ & $\mathrm{C} 7$ & $\mathrm{C} 8$ & $\mathrm{C} 9$ & $\mathrm{C} 10$ & $\mathrm{C} 11$ & $\mathrm{C} 12$ & $\mathrm{C} 13$ & Average \\
\hline $\begin{array}{c}\text { Precision } \\
(\%)\end{array}$ & 80 & 60 & 50 & 42.9 & 75 & 80 & 80 & 66.7 & 40 & 66.7 & 42.9 & 50 & 60 & 61.09 \\
Recall (\%) & 66.7 & 75 & 100 & 100 & 60 & 100 & 80 & 80 & 50 & 66.7 & 75 & 50 & 75 & 75.26 \\
\hline
\end{tabular}

Figure 13 shown the keyword in impressions and Finland churches that have been selected. The result of "historical" impression shown in Figure 14. 


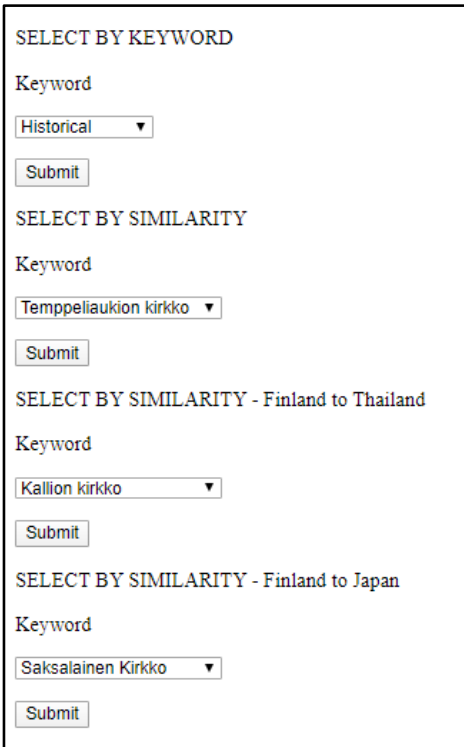

Figure 13. Impression and Finland churches

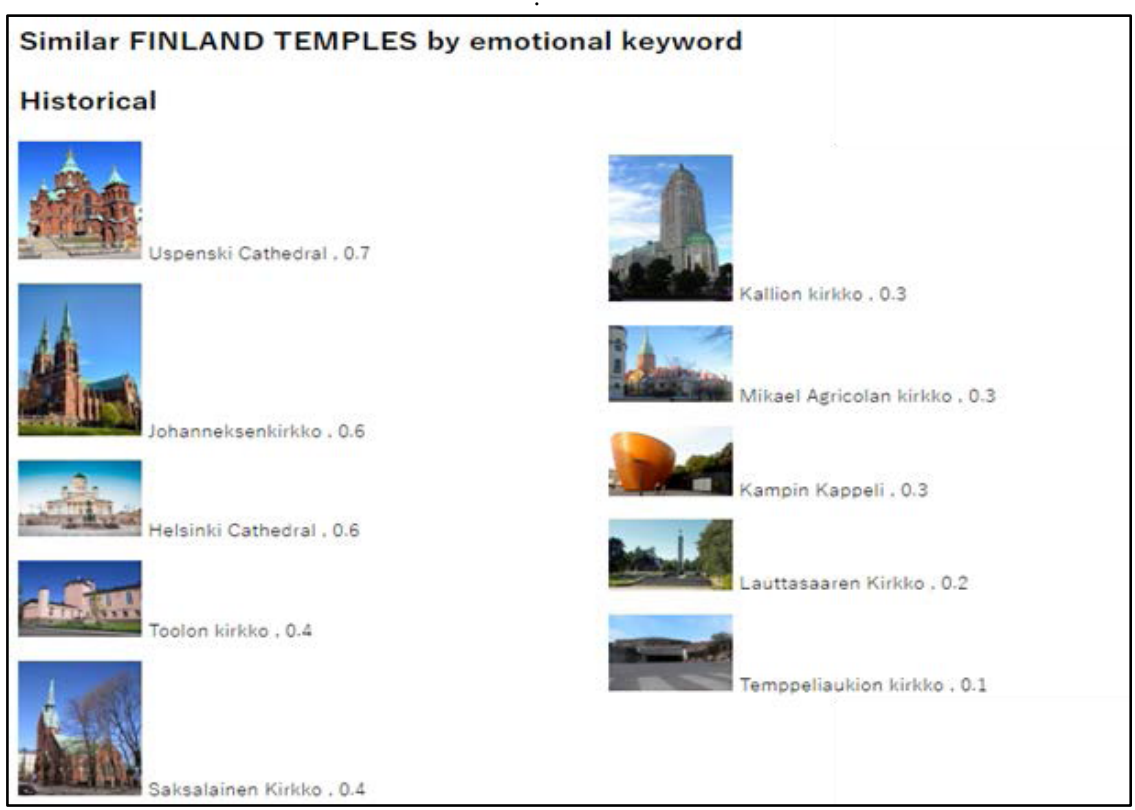

Figure 14. Finland Churches with "historical" impression.

Finland Churches that have the same impression with "Temppeliaukion kirkko" shown in Figure 15 and Figure 16 shows the Thailand temples that have the same emotion distance as "Kallion kirkko" and Figure 17 Show Japan temples that have the same impression with "Saksalainen kirkko". 


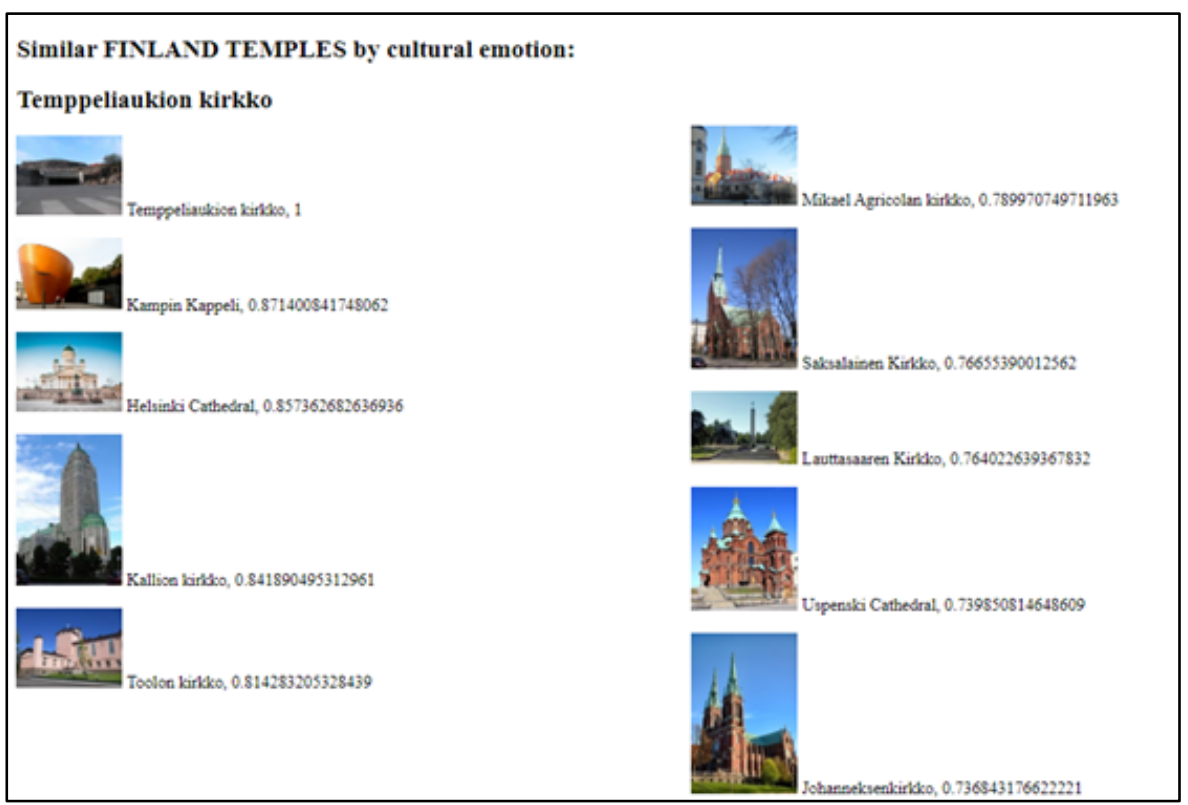

Figure 15. Finland churches similar with "Temppeliaukion kirkko".

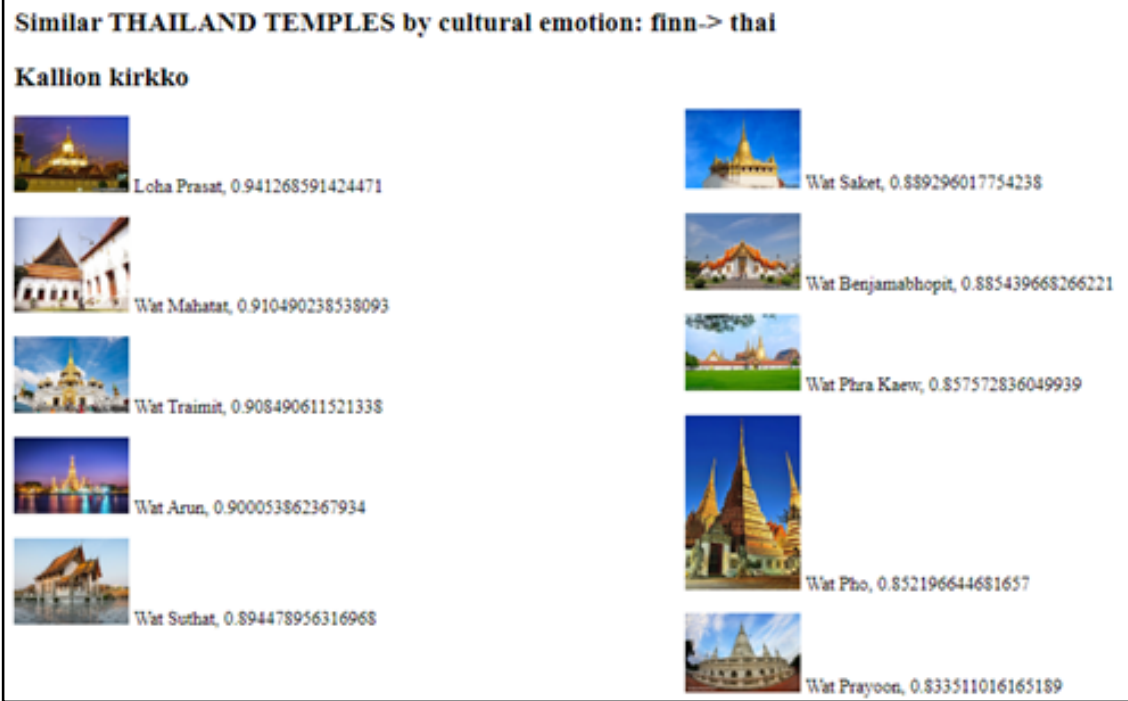

Figure 16. Thailand temples with emotion distance as "Kallion kirkko". 


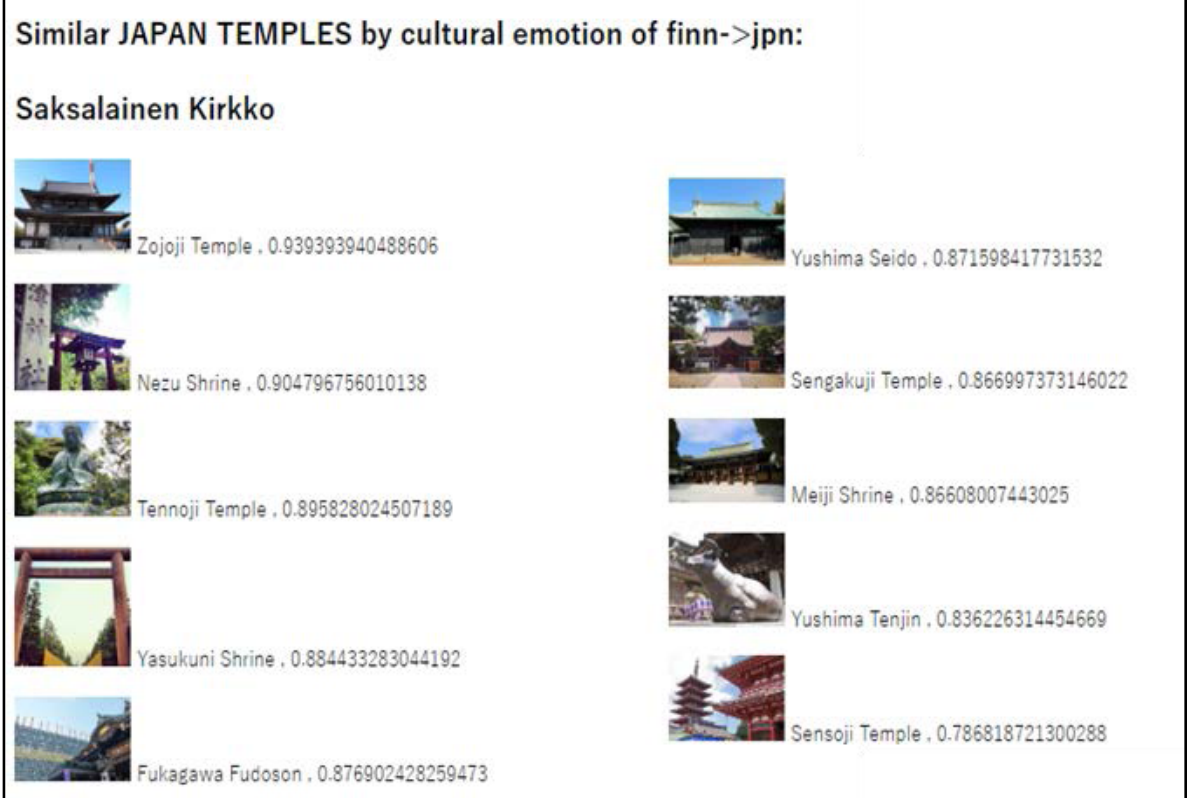

Figure 17. Japan temples with emotion distance as "Saksalainen kirkko".

\section{Conclusion}

This paper proposed the impression distance search system for cross-cultural religious tourism by presenting a variety of emotional responses from different cultures. We have described the main features of our research: (1) a culture-dependent metadata extraction method function for cross-cultural religious analysis and search. (2) a cross-cultural computing mechanism to represent the variety of emotional tourism interpretations from different religious-cultural with impression. As future work, we will extend the number of various application systems integration with symbolic essences database to improve cross-cultural tourism experiences with cultural color-analysis methodology [8]. Moreover we will using "Kansei" and semantic computing to new research for realizing knowledge sharing and mutual learning on global environmental issues.

\section{Acknowledgement}

This work is supported by Multimedia Database Laboratory (MDBL), Graduate School of Media and Governance, Keio University. We thank the MDBL members for their valuable comments and suggestions

\section{References}

[1] The World Tourism Organization. Tourism and the Sustainable Development Goals - Journey to 2030. US: UNWTO Publications; 2018. 114 p. 
[2] Kiyoki Y, Kitagawa T, Hayama T. A Metadatabase System for Semantic Image Search by a Mathematical Model of Meaning. SIGMOD Record. 1994 Jan 12;23(4):34-41.

[3] Y. Kiyoki and M. Kawamoto. Semantic Associative Search and space Integration Methods Applied to semantic Metrics for Multiple Medical Fields. Information Modelling and Knowledge Bases XVIII. 2007. p. $120-135$.

[4] Kitagawa, T. Kiyoki, Y. A mathematical model of meaning and its application to multidatabase systems. Proceedings of 3rd IEEE International Workshop on Research Issues on Data Engineering: Interoperability in Multidatabase system. 1993 April 19-20; Vienna, Austria: IEEE; c1993. p. 130-135.

[5] Kiyoki Y, Kitagawa T, Hitomi Y. A Fundamental Framework for Realizing Semantic Interoperability in a Multidatabase Environment. Integrated Computer-Aided Engineering. 1995 Jan;2(1):3-20.

[6] Potts C. From frequency to meaning: vector space models of semantics. Journal of Artificial Intelligence Research. 2010;37:141-188.

[7] Itabashi, Y., Sasaki, S. and Kiyoki, Y. Cross-cultural Image Computing with Multiple Color-Space Transformation. Journal of EMITTER. 2011;2(2):182-192. 